\title{
COVID-19 pandemic and its impact on emergency surgical services: A mini-review
}

\author{
Assama Riaz ${ }^{1,}{ }^{*}$, Dinali Obeysekera ${ }^{2}$ and Maya Itani ${ }^{3}$ \\ MUA (Medical University of the Americas), Medical School, C/O R3 Education Inc. 27 Jackson Road, Suite 302 Devens, \\ Massachusetts 01434, United States.
}

International Journal of Biological and Pharmaceutical Sciences Archive, 2021, 02(01), 001-006

Publication history: Received on 17 May 2021; revised on 26 June 2021; accepted on 29 June 2021

Article DOI: https://doi.org/10.53771/ijbpsa.2021.2.1.0054

\begin{abstract}
Background: The global COVID-19 spread is unquestionably one of the leading obstacles of every healthcare system, patient, and healthcare professionals. This leading pandemic has immeasurable effects on every healthcare domain, including surgical care, especially surgical demand for emergency settings.
\end{abstract}

Methods: An extensive data search was done from two leading databases Medline (PubMed) and Google Scholar, by using different keywords. The literature search was done both using individual and combination of keywords. All relevant data published from December 2019 to April 2021 were included and narratively analyzed.

Results: Thirteen articles were selected based on COVID-19 and its consequences on emergency Surgical Services including recommendations, guidelines, prioritization, and reporting of surgical case reduction.

Conclusion: This global COVID-19 pandemic had influenced all domains of surgery, including emergency surgery, which required urgent surgical attention. Training and multidisciplinary support are necessary for the surgical teams to perform in this critical situation.

Keywords: COVID-19; Surgery; Emergency surgery; Emergency

\section{Introduction}

The COVID-19 pandemic has markedly altered the healthcare practices globally, affecting over 3 million people in an escalating manner. The core goal of healthcare systems is to ensure transmission restriction of COVID-19 to noninfectious individuals providing all necessary patient care $[1,2]$. The COVID-19 pandemic was denounced as a global emergency by the World Health Organization (WHO) in March 2020 [3]. The medical surgery domain was one of the high-risk categories to expose the hospital workers to body fluids, especially blood and serum of COVID-19 positive patients. The Healthcare system neglected this concern because of its high-risk nature and substantial redirection of the medical system towards COVID-19 infection treatment and control [1,2]. The surgical department also faced the challenge of practicing strict infection control guidelines to avoid nosocomial infections and the spread of COVID-19 infection $[2,3]$.

Studies reported an extreme decline in surgical number because of COVID-19 with a total variation rate of -88.2 [4]. Another study reported the $-42.8 \%$ variation on admitting surgical cases and $-25.4 \%$ variation rate on emergency surgical procedures [5]. Center for Disease Control and Prevention (CDC), recommended postponing all elective surgeries [6] and many standard committees release their guidelines for true patient classification in terms of urgent

\footnotetext{
${ }^{*}$ Corresponding author: Assama Riaz

MUA Medical School, (Medical University of the Americas), C/O R3 Education Inc. 27 Jackson Road, Suite 302 Devens, Massachusetts 01434, United States.
}

Copyright $(2021$ Author(s) retain the copyright of this article. This article is published under the terms of the Creative Commons Attribution Liscense 4.0. 
care procedures and postponed cases of elective surgeries $[2,6]$. The surgical department should evaluate the case-bycase approach based on the standard indications and recommendations [2].

In low- and middle-income countries (LMICs) situation is more critical because of economic concerns and poor infection control practices [7]. Childbirth is also, a continual phenomenon and about 15\% of pregnancy cases need surgical help, including cesarean section, postpartum hemorrhage management, and obstructed labor [7].

This narrative study proposes to show the importance of different categories of surgical procedures, especially emergency surgeries that are observed unparalleled change and, at also, how rise can silently creep up in these change surgical practice guidelines.

\section{Methodology}

We created a mini systemic review of published scientific literature on COVID-19 and its impact on emergency surgical practices. We also analyze the important decision-making steps in surgical practices because of the COVID-19 pandemic. All relevant articles based on the search criteria from December 2019 to April 2021 were included.

\subsection{Data search and data extraction}

We selected two databases; Medline (PubMed) and Google Scholar for literature search by applying different keywords, such as COVID-19 and surgeries, Coronavirus and surgeries, COVID-19 and emergency surgeries, COVID-19 and emergency surgeries, and Corona virus and emergency surgeries. Keywords used both in combination or alone to cover all possible scientific literature searches.

\subsection{Study Inclusion Criteria}

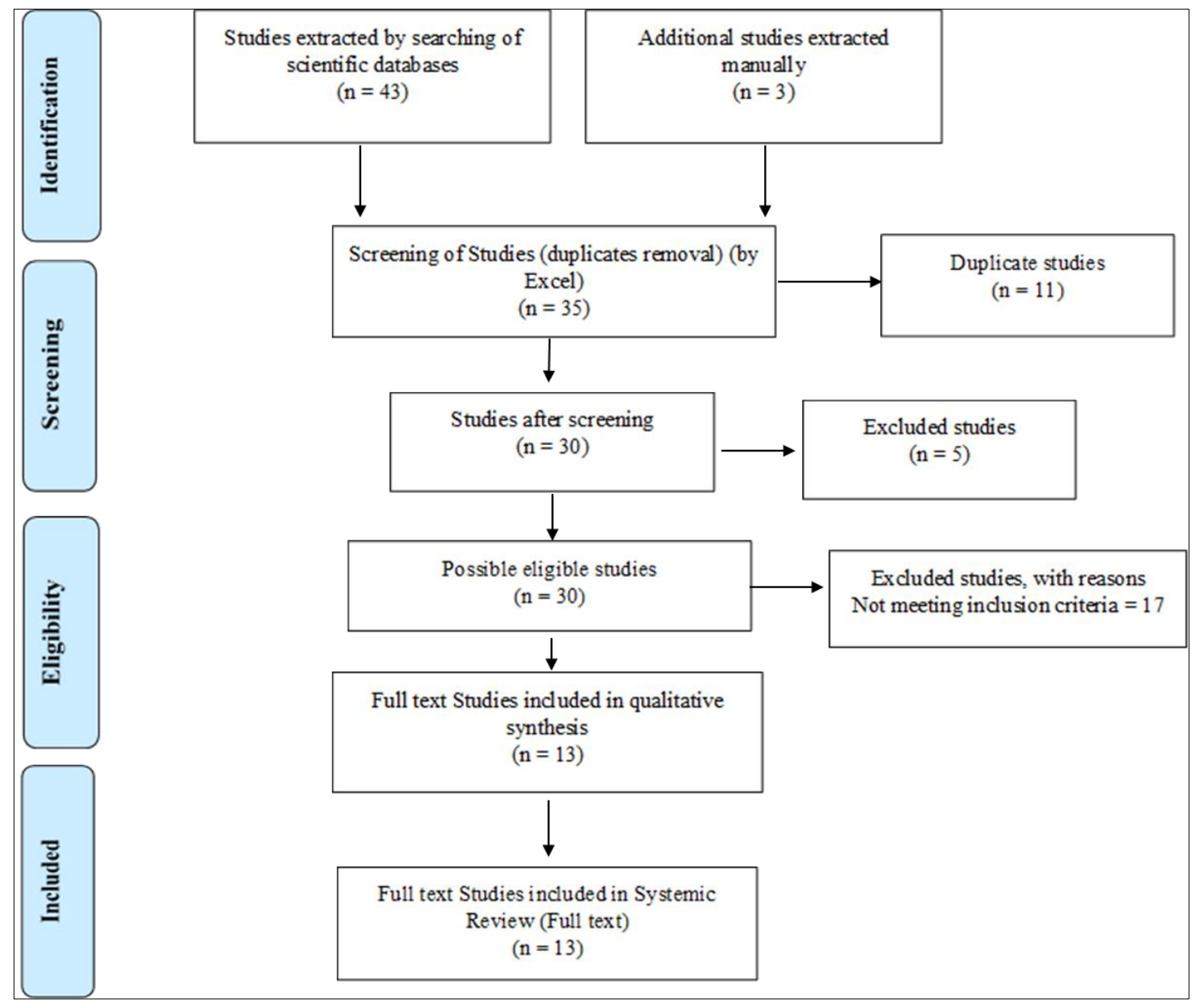

Figure 1 Outline of Study Selection by PRISMA schematic presentation 
All scientific original articles of all forms such as; editorials, recommendations, commentaries, and original data articles reported the "Emergency surgical services and COVID-19". We only included completely published articles of English language that reported all possible domains of emergency surgeries and their related impact to COVID-19.

"Preferred Reporting Items for Systematic Reviews and Meta-Analyses Protocols (PRISMA)" checklist followed for the data selection process [8]. Data segregation and selection is clarified by the PRISMA schematic chart (Fig. 1). Thirteen Studies fulfill the inclusion criteria for review writing, see Table 1.

\section{Results and discussion}

\subsection{Impact of emergency surgeries in low- and middle-income countries (LMICs)}

This study explores the potential impact of COVID-19 in LMICs and the possible surgical needs and related recommendations to reduce the damage and readiness to deal with future crises. This paper focuses on the need for essential emergency surgeries by empowering communities with the true exchange of information on preventive measures, optimization of surgical material, quality training of healthcare professionals to deal with COVID-19 crises. Future developments also need to address to handle such crises. This crisis helps LMICs to empower their communities and healthcare system to improve awareness among community and healthcare facilities [17] [19].

\subsection{True Identification and reporting of COVID-19 before surgical decision}

The COVID-19 positivity has an adverse effect on perioperative surgical outcomes. A definite diagnosis is very crucial for surgical decisions. The radiological method or molecular testing should perform the recommended preoperative screening by RT-PCR reverse transcriptase-polymerase chain reaction, according to the feasibility of the facility. This study suggested the recommendation of thorax computerized tomography (CT) or RT-PCR for every patient scheduled for emergency surgery [9].

\subsection{Recommendations and Guidelines for resumption of Surgeries}

This guideline gives the complete details of the surgical decision in the COVID-19 pandemic. COVID-19 Testing, Personal Protective Equipment (PPEs) requirement, Prioritization and categorization of patients, surgical plan and scheduling, Post-COVID-19 Issues, collection, and management of data, COVID-19 related infection control practices, assessment and mitigation of risks, and additional COVID-19 Related Issues. This guideline categorizes the Post surgical COVID-19 concerns into five stages including Phase I: Preoperative, Phase II: Immediate Preoperative, Phase III: Intraoperative, Phase IV: Postoperative, and Phase V: Discharge planning and Post Care [10].

\subsection{Recommendations and Guidelines of Surgical services requirements during COVID-19 Distress}

These recommendations were announced on March 30 th, 2020 to deal with COVID-19 crises and surgical concerns. These recommendations include the Rationing of Services, Procedural Considerations, and Practical Measures. The main reported concern was the prioritization of urgency and importance of case details before the surgical decision and suggested postponing all elective surgical and endoscopic cases. All unnecessary or clerical facility staff should allow staying home or working from home. All unnecessary hospital visits should be prohibited. Multidisciplinary team (MDT) effort is the key to success and should adapt to achieve better goals [11].

\subsection{Risk-stratification of Surgeries during COVID-19 pandemic}

This editorial gives a comprehensive detail of risk-stratification of surgical decision and provides a complete algorithm with duration, surgical signs, and case examples. The emergent surgical indications include severe life-threatening condition, Acute blood loss / exsanguination / extreme hemorrhage, level 1 Trauma, uncontrolled vascular injury, dissection of Aorta, C-section (Urgent/emergency), necrotizing fasciitis, Acute compartment syndrome, obstruction of Bowel, Peritonitis. These cases required $<1$ hour to address the concern and required urgent surgical decision [12].

\subsection{ESTES Recommendations}

These guidelines include that two Surgeons or consultants should critically analyze every acute case of a surgical decision to access the accurate risk of surgical proceeding or the possible alternative interventions. Any delay of intervention and its consequences must be considered before decision-making [13]. 


\subsection{COVID-19 and its impact on emergency surgical admissions and operative volumes}

This study reported a comparative pattern of emergency surgical admissions and operative volumes before and during the COVID-19 pandemic. The reduction rate of Total emergency procedures was $-25.4 \%$, Total operative procedures 29.8\%, Emergency Laparotomy $+40 \%$, Laparoscopic appendicectomy $-49 \%$, Open appendicectomy $+200 \%$, Incision and Drainage $-28.5 \%$, Total emergency endoscopy $-7.1 \%$, OGD -8.7\%, and Colonoscopy $-5.3 \%$ [5].

\subsection{COVID-19 red zone and emergency surgeries}

This study reported the reduction of surgeries by $86 \%$ which leads to tragic consequences in patients who need urgent care and emergent surgical requirement. The preventive measures should be implemented in every healthcare facility during COVID-19 are: Infection Control training of healthcare professionals ii) Limitations of surgical procedures and iii) Patient's screening [14] [20].

\subsection{Framework for surgical prioritization}

The study categorizes the surgical decision according to patient's need and urgency demand. The categories include

- Urgent: Surgical decision should be taken and performed as soon as issue addressed

- $\quad$ Less urgent: surgical deferral and over 30 days required to perform.

- Less Acute: postponed for 30-90 days

- According to case-by-case evaluation

Some reported categories with case examples are; Low or minimal risk surgery (no mucosal) includes; Thyroid and Parathyroidectomy, dissection of neck, skin/dermal cancer excision without involving eyes, mouth, nose, and sinus), Parotidectomy, and excision of Branchial cleft. High risk and critical transmucosal surgical procedures include; transoral surgery, resection of buccal, Glossectomy, etc., Lateral oropharyngectomy, Palatectomy, Oral cavity composite resection, maxillectomy, Rhinectomy, Laryngeal surgery, Vocal cord procedures, Partial/total laryngectomy, Transoral robotic surgery, and Direct laryngoscopy [15].

\subsection{Variation rate of emergency surgical experience}

This short correspondence reported the experience of Italian hospital and reported the variation rate of emergency surgical experience in the Pre-COVID-19 and COVID-19 Era. The Total\% variation rate of Overall access to the emergency department was $-88 \cdot 2$, General surgery $-83 \cdot 4$, Urology $-79 \cdot 9$, Orthopaedics $-92 \cdot 9$, Maxillofacial surgery $-95 \cdot 0$, Ear, nose \& throat $-74 \cdot 1$, Neurosurgery $-94 \cdot 2$, Cardiac surgery $-75 \cdot 0$, Thoracic Surgery $+150 \cdot 0$, and Vascular surgery $-91 \cdot 2[4]$.

\subsection{Colorectal surgery and impact of COVID-19}

This correspondence reported the major reduction of surgical procedures globally because of COVID-19. All non-urgent surgical procedures postponed because of less priority, delay in colorectal malignancies, might lead to serious outcomes [16].

\subsection{A retrospective study on emergency general surgery}

This study reported the reduction of unnecessary nonemergency surgeries and admissions but not found any significant reduction in patients' urgent consultations and emergency surgeries. The reported cases include Colon Appendectomy or resection of small bowel, Hartmann surgical resection, Iridectomy, repairing of GIS perforation, Total gastrectomy, Hernia repair or Herniography, and laparotomy. All patients were diagnosed by Nonspecific abdominal pain, acute appendicitis, acute hepatobiliary infections, acute mesenteric ischemia, GIS perforations, Intestinal obstructions, and GIS bleeding [17].

\subsection{Surgical patients and their management in emergency settings}

This is the most recent expert recommendation by WSES on emergency surgical care during the COVID-19 pandemic. This study categorizes the emergency surgeries' and their possible management according to the critical condition grouping and time to address. According to the TACS (Timings of the Acute Care Surgery), there were six groups. The urgent care groups were "Immediate Surgical decision" because of acute bleeding conditions, and "within one hour", group that required surgical procedure within an hour, such as; acute incarcerated hernia. All other conditions should be managed by therapeutic support and schedule for the surgical procedures if required according to the severity, De Simone, et al., 2021 [18]. 


\section{Conclusion}

Delivering and resuming surgical practices as similar to the before COVID-19 pandemic era has countless challenges for patient, patient's career, healthcare facilities, surgeons, and all healthcare workers. However, all hospital facilities and healthcare professionals should be trained according to new and updated infection control guidelines and implemented to perform critical surgeries, which won't be postponed.

\section{Compliance with ethical standards}

\section{Acknowledgments}

We would like to thank our institute's support for conducting research writing.

\section{Disclosure of conflict of interest}

No conflict of interest reported.

\section{References}

[1] Huda F, Kumar P, Singh SK, Agrawal S, Basu S. COVID-19 and surgery: Challenging issues in the face of new normal - A narrative review. Ann Med Surg (Lond). Dec 2020; 60: 162-167.

[2] Soltany A, Hamouda M, Ghzawi A, Sharaqi A, Negida A, Soliman S, Benmelouka AY. A scoping review of the impact of COVID-19 pandemic on surgical practice. Ann Med Surg (Lond). 9 Jul 2020; 57: 24-36.

[3] World Health Organization (Who), Corona Virus Disease 2019 (COVID-19) Situation Report-51, Available at (March 11 2020):, Last accessed. 12 May 2020

[4] Castagneto-Gissey L, Casella G, Russo MF, Del Corpo G, Iodice A, Lattina I, Ferrari P, Iannone I, Mingoli A, La Torre F. Impact of COVID-19 outbreak on emergency surgery and emergency department admissions: an Italian level 2 emergency department experience. Br J Surg. Sep 2020; 107(10): e374-e375.

[5] O'Connell RM, Khan MA, Amir M, Bucheeri M, Khan W, Khan IZ, Barry KM. The impact of COVID-19 on emergency general surgery admissions and operative volumes: A single centre experience. Surgeon. 19 Nov 2020.

[6] American college of Surgeons, COVID-19: Elective Case Triage Guidelines for Surgical Care. 24 March 2020.

[7] Ma X, Vervoort D, Reddy CL, Park KB, Makasa E. Emergency and essential surgical healthcare services during COVID-19 in low- and middle-income countries: A perspective. Int J Surg. Jul 2020; 79: 43-46.

[8] Swartz MK. The PRISMA statement: a guideline for systematic reviews and meta-analyses. J Pediatr Health Care 2011; 25: 1-2.

[9] Hogan A. COVID-19 and emergency surgery. Br J Surg. Jun 2020; 107(7): e180.

[10] American College of Surgeons, American Society of Anesthesiologists, Association of periOperative Registered Nurses, and American Hospital Association, “Joint Statement: Roadmap for Resuming Elective Surgery after COVID-19 Pandemic. 2020; 9

[11] Pryor A. SAGES and EAES recommendations regarding surgical response to COVID19 crisis,

[12] Stahel PF. How to risk-stratify elective surgery during the COVID-19 pandemic? Patient Saf Surg. 31 Mar 2020; 14: 8 .

[13] Coimbra R, Edwards S, Kurihara H. et al. European Society of Trauma and Emergency Surgery (ESTES) recommendations for trauma and emergency surgery preparation during times of COVID-19 infection. Eur J Trauma Emerg Surg. 2020; 46: 505-510.

[14] Patriti A, Eugeni E, Guerra F. What happened to surgical emergencies in the era of COVID-19 outbreak? Considerations of surgeons working in an Italian COVID-19 red zone. Updates Surg. Jun 2020; 72(2): 309-310.

[15] Topf MC, Shenson JA, Holsinger FC, Wald SH, Cianfichi LJ, Rosenthal EL, Sunwoo JB. Framework for prioritizing head and neck surgery during the COVID-19 pandemic. Head Neck. Jun 2020; 42(6): 1159-1167.

[16] Lisi G, Campanelli M, Spoletini D, Carlini M. The possible impact of COVID-19 on colorectal surgery in Italy. Colorectal Dis. Jun 2020; 22(6): 641-642 
[17] Tarim IA, Derebey M, Özbalci GS, Özşay O, Yüksek MA, Büyükakıncak S, Bircan R, Güngör BB, Başoğlu M. The impact of the COVID-19 pandemic on emergency general surgery: a retrospective study. Sao Paulo Med J. 29 Jan 2021.

[18] De Simone, B., Chouillard, E., Sartelli, M. et al. The management of surgical patients in the emergency setting during COVID-19 pandemic: the WSES position paper. World J Emerg Surg. 2021; 16: 14.

[19] George AS, Mehra V, Scott K, Sriram V. Community Participation in Health Systems Research: A Systematic Review Assessing the State of Research, the Nature of Interventions Involved and the Features of Engagement with Communities. PLoS One. 23 Oct 2015; 10(10): e0141091.

[20] Organization WHO (2014) World Health Organization (WHO), infection prevention and control of epidemic- and pandemic-prone acute respiratory infections in health care. 1-156. 